\title{
Semiflexible magnetic filaments near attractive flat surfaces: Langevin Dynamics study.
}

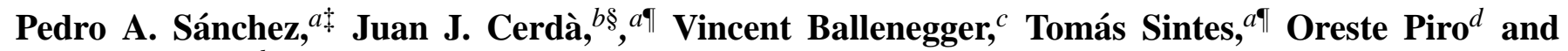 \\ Christian Holm ${ }^{b}$
}

\author{
Received Xth $X X X X X X X X X X 20 X X$, Accepted Xth $X X X X X X X X X 20 X X$ \\ First published on the web Xth $X X X X X X X X X X 200 X$ \\ DOI: 10.1039/b000000x
}

The adsorption of stiff magnetic filaments close to an attractive surface is studied thoroughly via extensive Langevin Dynamics simulations (LD). Magnetic filaments are represented by a coarse-grained bead-spring model where each bead bears a point dipole located at its center and the excluded volume interaction is introduced via a soft-core repulsive potential. We find strong evidence for the existence of two transitions as the temperature is lowered. First, the system undergoes a continuum phase transition from the non adsorbed to the adsorbed state. This transition is followed by a second structural transition that takes place when the filaments are already adsorbed. The adsorption transition is found to be very similar to the one observed for stiff non-magnetic polymer chains [Sintes et al., Macromolecules 2001, 34, 1352-1357] where the chain bending interaction plays a similar role as the magnetic component of the present case. However, the tendency of the magnetic chains to stretch is reversed by a further reduction in temperature and chains tend to form closed adsorbed loops leading to a second structural transition. A representation of phase diagram for the adsorption of magnetic filaments is determined here for the first time. We also present a novel way to determine the temperature at which the chain is adsorbed that is based on the analysis of the change in the number of trains, tails and loops developed by the polymer chain during the adsorption process.

\section{Introduction}

The synthesis of artificial magnetic filaments by mutually linking particles with magnetic properties has set the path towards the construction of supramolecular magnetic polymers. Initially, Furst et al. ${ }^{1,2}$ reported in seminal papers the use of micron-sized magnetic-filled paramagnetic latex beads as monomers to form magnetic chains. Further progresses was done by Dreyfus et al. ${ }^{3}$ using DNA to link micron-sized paramagnetic beads. The first steps towards the assembly of magnetic filaments using sub-micrometric particles $(0.5-0.8 \mu \mathrm{m})$ where reported by Tabata et al. ${ }^{4}$. The synthesis of chains with lengths in the range 30 to $50 \mu \mathrm{m}$ either in solution or attached to a surface was reported by Singh et al. ${ }^{5,6}$ and Toonder et al. ${ }^{7}$. On the other hand, the synthesis of arrays of long magnetic filaments (from 1 to $200 \mu \mathrm{m}$ ) with length-to-monomer-size ra-

a Instituto de Física Interdisciplinar y Sistemas Complejos, IFISC (CSICUIB). Universitat de les Illes Balears. E-07122 Palma de Mallorca, Spain.

${ }^{b}$ Institute for Computational Physics. Universität Stuttgart. 70569 Stuttgart, Germany.

${ }^{c}$ Institut UTINAM. Université de Franche-Comté, CNRS. 16, route de Gray, 25030 Besançon cedex France.

${ }^{d}$ Departamento de Física. Universitat de les Illes Balears. E-07122 Palma de Mallorca, Spain.

$\ddagger$ E-mail: pedro@ifisc.uib-csic.es

$\S$ E-mail: joan@ifisc.uib-csic.es

I E-mail: tomas@ifisc.uib-csic.es tios up to 125 times was obtained by Evans and co-workers ${ }^{8}$. Further technological improvements have been possible and the use of nanoparticles with sizes below 50 $\mathrm{nm}$ to form mesoscopic one-dimensional structures has become a reality ${ }^{9-15}$. Very recently, Benkoski et al. ${ }^{16}$ have reported the optical visualization of small ferromagnetic particles $(23.5 \mathrm{~nm})$ organizing into microscopic one-dimensional chains which can be assembled into dense filament arrays just using magnetic interactions on surfaces without the resource of templates or multiple fabrication steps.

The continuous progress in the size reduction of the magnetic particles and the improvement of the links between them has brought us to the onset of the production of assemblies of chains that resemble magnetic polymers but at the scale of tenths of nanometers. Although the exact range may depend strongly on the type of materials used, magnetic cores with sizes between 10 and 100 $\mathrm{nm}$ can remarkably have permanent dipolar moment at room temperature in the absence of external magnetic fields, because they basically form a single ferromagnetic domain ${ }^{17}$. On the other hand, cores smaller than $10 \mathrm{~nm}$ are expected to exhibit a superparamagnetic behavior due to the relaxation processes, whereas cores larger than $100 \mathrm{~nm}$ show paramagnetic properties due to the formation of multiple domains ${ }^{18}$. The permanent magnetic properties of these filaments contrast with those of chemical magnetic polymers that are only manifested at very low tempera- 
tures $(\mathrm{T}<100 \mathrm{~K})^{19,20}$.

Permanent magnetic filaments are very interesting from the point of view of technological applications because of their potential use in magnetic memories, chemical and pressure nanosensors, generation of unique patterns able to provide watermarks to authenticate cards and other documents, etc. Therefore, since many of such applications involve the interaction of magnetic filaments with a surface, the detailed understanding of the properties of such interaction is crucial for the technological developments. On the other hand, from a more general point of view, the study of the behavior of magnetic filaments near surfaces addresses the more general and very interesting basic question as of how non-isotropic interactions influence the adsorption transition of polymer chains.

To our best knowledge, the characterization of the properties of magnetic filaments has been so far limited to the study of the behavior of single magnetoresponsive filaments in the bulk, i.e. far away from any surface ${ }^{18,21-25}$, the study of magnetic filaments as micro-propellers (microswimmers) in the presence of non steady magnetic fields ${ }^{26-28}$, and the study of the equilibrium shapes of twisted magnetic filaments ${ }^{29}$. A thorough study of the properties of permanent magnetic filaments close to an interacting surface has not been performed yet, though several works have already focused on the confinement effects near surfaces in dipolar systems?

In the present work we characterize the adsorption process of stiff or semi-flexible permanent magnetic filaments near an attractive surface and compare their behavior to that observed for non-magnetic chains in the same conditions, a subject that has experimented a substantial progress during the last decades. For instance, the transition from a desorbed to an adsorbed state in good $^{30-45}$ and poor ${ }^{43,46-58}$ solvent conditions has been investigated in detail. In the case of semi-flexible ideal chains Khokhlov et al ${ }^{59}$ presented an analytical solution proving that the adsorption process is a second-order phase transition in the limit of infinite chain length. The influence of the chain stiffness on adsorption has also been studied using coarse-grained numerical simulations ${ }^{37,60-62}$ and the phase diagram of systems with competing forces have been numerically determined ${ }^{48-57,63-65}$. For neutral polymers, the conformations of adsorbed phases have been studied by Möddel et al. ${ }^{66}$ using multicanonical computer simulations. In addition to the main phases of adsorbed and desorbed conformations several other phase transitions have been observed, as for example, freezing transitions between energy-dominated crystalline low-temperature structures and globular entropydominated conformations ${ }^{67,68}$. The case of the adsorption of charged polymers onto surfaces has also received considerable attention (see Dobrynin ${ }^{69}$ for an updated review). Very recently, Monte Carlo simulations by Rissanou et al. ${ }^{70}$ studied the coil-globule transition near an attractive surface and the adsorption of random copolymers from a melt onto a solid surface was analyzed by Klos et $\mathrm{al}^{71}$.

In this paper we report our investigations on the influence of the magnetic interactions on the process of adsorption of stiff polymer chains by an attractive surface. We show the results of extensive Langevin Dynamics simulations in quasi-two dimensional systems using a coarse bead-spring chain model for the filaments, that allowed us to thoroughly review the effects of the temperature, the strength of the magnetic interaction, the stiffness and the chain length. On one hand, it is known that in ferrofluids the magnetic interaction favors the arrangement of particles dipoles in a head to tail configuration inducing the creation of structures similar to chains ${ }^{72}$. This would let us expect that the same interactions should contribute to increase the stiffness of a chain composed of bonded magnetic particles. On the other hand, it is also known that ferrofluid particles tend to form annular structures ${ }^{72}$ that were found to be the ground state of the system in quasi-two dimensional geometries $^{73}$. We then expect that the magnetic interaction have an influence on the chain morphology that is very different from the one owed to the stiffness. However, it is not trivial to anticipate what the effect of the dipolar interaction will be on semi-flexible magnetic chains close to an attractive surface and the clarification of this have been one of the main purposes of our labor.

In Section 2 we describe the numerical model and the details of our simulation exercises, in Section 3 we present and discuss our results and finally a summary and a discussion of the conclusions is presented in Section 4.

\section{Numerical Model}

To investigate the behavior of stiff magnetic filaments close to an attractive surface in the limit of infinite dilution we perform simulations with a model of a single magnetic chain placed in an constrained open space unbounded along the $x-y$ plane but limited in the $z$ direction by two impenetrable walls located at $z=0$ and $z=50 \sigma$, where $\sigma$ is the monomer diameter.

The magnetic filaments are modeled in the simplest possible way as a bead-spring semiflexible chain of $N \in[20,300]$ monomers constituted by individual magnetic beads. Each bead has a point dipole located at its center. The beads are subject to a mutual steric repulsion described by a truncatedshifted Lennard-Jones potential (tsLJ) also known as a WeeksChandler-Andersen potential ${ }^{74}$ :

$$
U_{t s L J}(r)= \begin{cases}U_{L J}(r)-U_{L J}\left(r_{c u t}\right), & r<r_{\text {cut }} \\ 0, & r \geq r_{\text {cut }}\end{cases}
$$

where $U_{L J}(r)$ is the standard Lennard-Jones:

$$
U_{L J}(r)=4 \varepsilon_{e}\left[\left(\frac{\sigma}{r}\right)^{12}-\left(\frac{\sigma}{r}\right)^{6}\right] .
$$


For simplicity in our simulations we use reduced units so that $\sigma=1$ and $\varepsilon_{e}=1$. The cutoff radius $r_{\text {cut }}$ for the tsLJ potential is set to $r_{\text {cut }}=2^{1 / 6} \sigma$ in order to warrant that monomers repel each other with a soft-core potential. A finite extensible nonlinear elastic (FENE) potential is used to provide the connectivity between adjacent monomers in the chain:

$$
U_{\text {fene }}(r)=\frac{-K_{f} r_{\max }^{2}}{2} \ln \left[1-\left(\frac{r}{r_{\max }}\right)^{2}\right]
$$

with $K_{f}=30 / \sigma^{2}$, and $r_{\max }=1.5 \sigma$. It should be pointed that attaching the spring between beads to the center of the spheres rather than to points on the surface constitutes a first approach to the experimental situation in which the contribution of the rotations of the particle to the stretching of the bond is neglected.

The chain stiffness is introduced by way of the following bending potential ${ }^{61,62}$ :

$$
U_{B}=\sum_{\theta} \frac{\kappa}{2}(1+\cos \theta)^{2}
$$

where $\theta$ is the bond angle between any three consecutive sites in the chain and $\kappa$ is the bending constant. In the absence of magnetic interactions, the stiffness parameter $\kappa$ is directly related to the chain persistence length which can be experimentally measured by scattering techniques in real polymer chains ${ }^{75-77}$. We have explored chain stiffness degrees within the range of $\kappa \in[0,30]$, where $\kappa=0$ corresponds to a completely flexible chain.

The attractive surface is located at $z=0$ and monomers interact with the neutral and non-magnetic surface via a truncated-shifted 9-3 Lennard-Jones ${ }^{78}$ potential:

$$
U_{s}(z)= \begin{cases}U_{9-3}(z)-U_{9-3}\left(z_{\text {cut }}\right), & z<z_{\text {cut }} \\ 0, & z \geq z_{\text {cut }}\end{cases}
$$

where

$$
U_{9-3}(z)=4 \varepsilon_{s}\left[\left(\frac{\sigma}{z}\right)^{9}-\left(\frac{\sigma}{z}\right)^{3}\right]
$$

is a potential obtained by integration of the original LennardJones potential over an infinite flat surface. For simplicity we have taken $\varepsilon_{s}=\varepsilon_{e}=1$. The cut-off is set to $z_{\text {cut }}=2.5 \sigma$. A second purely repulsive neutral and non-magnetic surface is located at $z=50 \sigma$ to prevent the chain to reach too far from the adsorbing surface in order to save computational time during the equilibration period. Several tests have been performed locating the repulsive wall at $z>>50 \sigma$ in order to check that its presence does not bias the adsorption profile of the magnetic chain at the attractive surface. The monomers interact with the repulsive wall via a truncated shifted $\mathrm{LJ}$ potential like in Eq. 1 with $r=50-z$ and $z_{\text {cut }}=2^{1 / 6} \sigma$.
The magnetic nature of the filament is simulated by attaching a point dipole of permanent magnetic moment $\boldsymbol{\mu}$ at the center of each bead whose direction can freely rotate in the $3 \mathrm{D}$ space. The interaction between point dipoles is given by the potential:

$$
U^{d i p}\left(\boldsymbol{r}_{i j}\right)=\frac{\boldsymbol{\mu}_{i} \cdot \boldsymbol{\mu}_{j}}{\left|\boldsymbol{r}_{i j}\right|^{3}}-\frac{3\left[\boldsymbol{\mu}_{i} \cdot \boldsymbol{r}_{i j}\right]\left[\boldsymbol{\mu}_{j} \cdot \boldsymbol{r}_{i j}\right]}{\left|\boldsymbol{r}_{i j}\right|^{5}},
$$

where $\mathbf{r}_{i j}=\mathbf{r}_{i}-\mathbf{r}_{j}$ is the displacement vector between particles $i$ and $j$. The intensity of the dipolar interaction $\mu^{2}$ is proportional to the maximum dipolar energy between two dipoles and can be related to the experimental value by $\mu^{2}=$ $\mu_{e}^{2} /\left(\varepsilon_{e} \sigma_{e}^{3}\right)$, where $\sigma_{e}$ and $\varepsilon_{e}$ are respectively the range and the strength of the interacting potential and $\mu_{e}$ is the experimental dipolar magnetic moment. In general $\mu^{2}$ depends on the composition and size of the particles but it is typically found that $\mu^{2}<10$. The reduced temperature $T$ is defined as $T \equiv k_{B} T_{e} / \varepsilon_{e}$, where $T_{e}$ is the experimental temperature. A dimensionless dipolar coupling parameter $\lambda$ is often used to characterize the strength of the dipolar interaction. $\lambda$ is defined as half the ratio of the magnetic energy of two dipoles separated by one particle diameter and oriented head to tail, to the thermal energy. In reduced units we have $\lambda=\mu^{2} / T$.

The energy due to magnetic interactions are calculated by direct summation over all pairs of particles. In spite of being algorithmically $O\left(N^{2}\right)$ this is the fastest way to compute it because of the relatively small number of particles considered $N \in[20,300]$ and the fact that boundary conditions are not periodic. More complex methods involving Ewald sums or based on Fast Fourier Transform ${ }^{79}$ need periodic boundary conditions and require special care when one deals with magnetic chains in the limit of infinite dilution. Moreover, in all these advanced methods, that are very useful for large systems, the computation of the interactions is always approximated within a certain degree of accuracy in contrast with the direct summation that is exact.

The translational and rotational Langevin equations of motion of particles $i$ are given by ${ }^{80}$ :

$$
\begin{aligned}
M_{i} \frac{d \mathbf{v}_{i}}{d t} & =\boldsymbol{F}_{i}-\Gamma_{T} \boldsymbol{v}_{i}+\boldsymbol{\xi}_{i}^{T} \\
\boldsymbol{I}_{i} \cdot \frac{d \boldsymbol{\omega}_{i}}{d t} & =\boldsymbol{\tau}_{i}-\Gamma_{R} \boldsymbol{\omega}_{i}+\boldsymbol{\xi}_{i}^{R}
\end{aligned}
$$

where $\boldsymbol{F}_{i}$, and $\boldsymbol{\tau}_{i}$ are respectively the total force and torque acting on the particle $i, M_{i}$ and $\boldsymbol{I}_{i}$ are its mass and inertia tensor while $\Gamma_{T}$ and $\Gamma_{R}$ are the translational and rotational friction constants. $\xi_{i}^{T}$ and $\xi_{i}^{R}$ are a Gaussian Random force and torque, each of zero mean and satisfying usual fluctuationdissipation relations. Finally, the reduced time $t$ is given by $t=t_{e} \sqrt{\varepsilon_{e} /\left(M_{e} \sigma_{e}^{2}\right)}$, where $M_{e}$ and $t_{e}$ are the mass of the bead and the measured time respectively. 
For equilibrium simulations, the values of the mass, the inertia tensor, as well as friction constants $\Gamma_{T}$, and $\Gamma_{R}$ are irrelevant. The particle mass is chosen to be $M=1$ and we take the inertia tensor to be the identity matrix in order to ensure isotropic rotations $\boldsymbol{I}=\mathbf{1}$. We have chosen $\Gamma_{T}=1$ and $\Gamma_{R}=3 / 4$ because we observed that these values produced a conveniently fast relaxation to equilibrium ${ }^{72,81}$. We have also set the reduced time step to $\delta t=5 \cdot 10^{-3}$.

The simulation starts by placing the filament in the bulk region with its first bead located in the interval $z \in(\sigma, 5 \sigma)$. This choice guarantees that the chain is sufficiently close to the attracting surface to avoid the unnecessary computational time that it would spend just diffusing in the bulk. The remaining monomers are positioned using a self-avoiding random walk scheme with an overlap radius of $0.9 \sigma$. The chain is preequilibrated for a period of 50000 steps using a sequence of increasing time steps until the final value $\delta t$ is reached. Subsequently, the chain is equilibrated for a period of $2 \cdot 10^{6} \delta t$ to ensure that the results have lost memory of starting conditions. After the equilibration period, the system is sampled at intervals of $4000 \delta t$ for another period of $2 \cdot 10^{6} \delta t$ and to get further assurance that the results do not depend on the initial conditions we take the average over 70 independent runs for each monitored temperature. The simulations have been performed using the package ESPResSo ${ }^{82}$.

\section{Results and discussion}

We have performed extensive numerical simulations of the adsorption process of stiff magnetic filaments by a flat homogeneous surface for chain lengths varying in the range $N \in$ $[20,300]$. The strengths of the magnetic point dipole of the monomers considered were within the range $\mu^{2} \in[1,10]$.We present here the results for filaments with a stiffness $\kappa=10$ since the different explored values of $\kappa \leq 30$ ) show no qualitatively difference. The limit of persistence lengths similar or larger than the chain contour length was not studied in this work. The range of temperatures considered was $1 / T \in$ $[0.25,4]$. At high temperatures in the order of $1 / T \sim 0.25$ all chains are completely desorbed whereas at low temperatures $1 / T \sim 4$ they are fully adsorbed.

A direct inspection of equilibrium chain conformations shown in Figure 1, evidences the existence of three possible different phases in the system. At high temperatures, the chain is desorbed and exhibits the shape of a random coil with little order in the orientation of their dipoles (Fig. 1(a)). As the temperature is lowered the filament is adsorbed onto the surface and dipoles align with the chain backbone which tends to adopt more stretched conformations (Fig. 1(b)). Except for the presence of the dipoles, this situation corresponds to the well known behavior observed for non-magnetic semiflexible polymers whose adsorption process undergoes a continuum

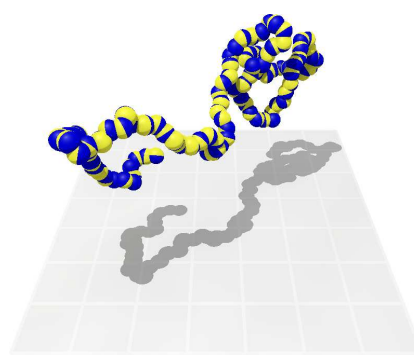

(a)

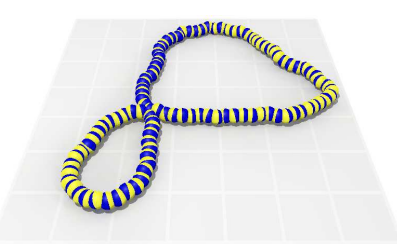

(c)

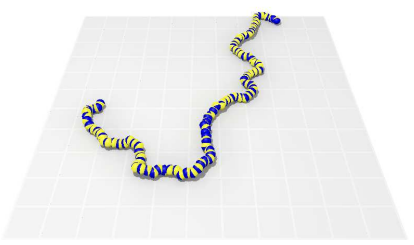

(b)

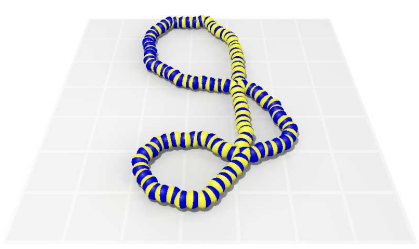

(d)
Fig. 1 Snapshots of equilibrium conformations of a magnetic filament near a flat homogeneous attractive surface for $N=100$, $\kappa=10$ and $\mu^{2}=3$ at different temperature values. Filament beads are depicted as two-colored spheres with the two colors representing the magnetic dipole orientation. (a) $1 / T=0.5$, (b) $1 / T=1.0$, (c) $1 / T=3.0$, (d) $1 / T=4.0$.

phase transition. Nonetheless, below a certain characteristic temperature, that depends on the chain parameters, the tendency to stretch is reversed and the equilibrium chain conformations are characterized by the presence of closed structures in the adsorbed state, forming planar loops with a smooth curvature and an almost perfect alignment of the dipoles along the chain (Fig. 1(c) and Fig. 1(d)). This behavior suggests the existence of a second structural transition that is a consequence of the competing interactions in the system. A further reduction in temperature apparently induces an increase in the number of loops (Fig. 1(d)). However, it is difficult to conclude whether the equilibrium ground state of the adsorbed magnetic filament follows this observation. Simulations at $1 / T>>4$ with conventional Langevin Dynamics (LD) take a prohibitive time to obtain an adequate statistics and more sophisticated techniques are required.

We also found evidences of a more complex behavior in the limit of very strong magnetic interactions at low temperatures. Figure 2(b) depicts the shape of a typical filament structure at $1 / T=4.0$ and $\mu^{2}=10$. In this range, entropy competes with strong secondary dipole-dipole interactions between beads which are far in the chain sequence though close in space leading to an effective attraction among those beads, and therefore more compacted conformations adsorbed on the surface result. This behavior is similar to the one observed in neutral polymer chains immersed in a poor solvent near an at- 


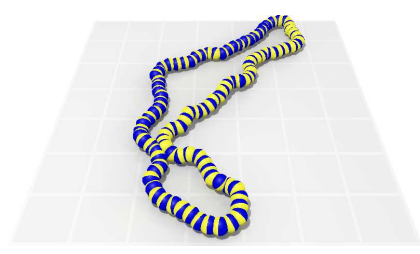

(a)

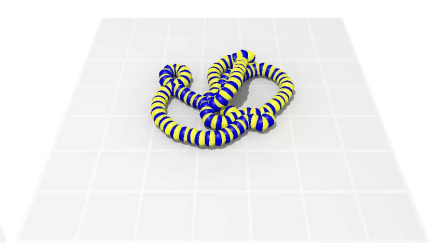

(b)
Fig. 2 Equilibrium chain conformations obtained at low temperatures $(1 / T=4.0)$. (a) A fully flexible filament $(\kappa=0)$ for $N=100$ and $\mu^{2}=3.0$ displays a more corrugated shape in comparison with Figs. 1(c) and 1(d); (b) Effect of high magnetization values $\left(\mu^{2}=10\right)$ on the chain conformation $(N=100, \kappa=10)$.

tractive surface in which an adsorbed collapsed phase is identified $^{52}$. The role of the chain stiffness is illustrated in Fig. 2(a) where a fully flexible chain displays a more corrugated shape in comparison with the analogous stiff chain.

As mentioned before, dipoles tend to align locally with the filament structure as the temperature is reduced. In order to quantify the degree of alignment we define a bond-dipole local orientation parameter as:

$$
\langle A\rangle=\frac{1}{N-2}\left\langle\sum_{i=2}^{N-1}\left|\hat{\boldsymbol{r}}_{i-1, i+1} \cdot \hat{\boldsymbol{\mu}}_{i}\right|\right\rangle,
$$

where $\langle\ldots\rangle$ means the average over the different collected chain conformations and runs at a given temperature $T$, $\hat{\boldsymbol{r}}_{i-1, i+1}$ is the unitary vector director that joins the center of the particles $i-1$ and $i+1$, and $\hat{\boldsymbol{\mu}}_{i}$ is the unitary dipole moment associated to bead $i$. When $\langle A\rangle \rightarrow 1$ a perfect local alignment of the chain with the dipoles is expected. The results for $N=100$ and $\kappa=10$ are shown in Figure 3. The local ordering tends to increase as the temperature is reduced until $\langle A\rangle$ reaches a plateau around $\langle A\rangle \sim 0.95$. The local alignment is never perfect due to the small but necessary curvature needed to form the closed structures that develop at small temperatures (see Fig.1). The best local alignment of the dipoles with the chain backbone is achieved at higher temperatures for increasing dipolar strength.

In the next two sections we will characterize the undergoing continuum phase transition of the magnetic filament towards the adsorbed state, and the induced structural transition from open stretched chains to close folded configurations expected to appear at small temperatures.

\subsection{The adsorption transition}

The adsorption transition in polymeric systems can be characterized using observables like the mean normalized adsorption

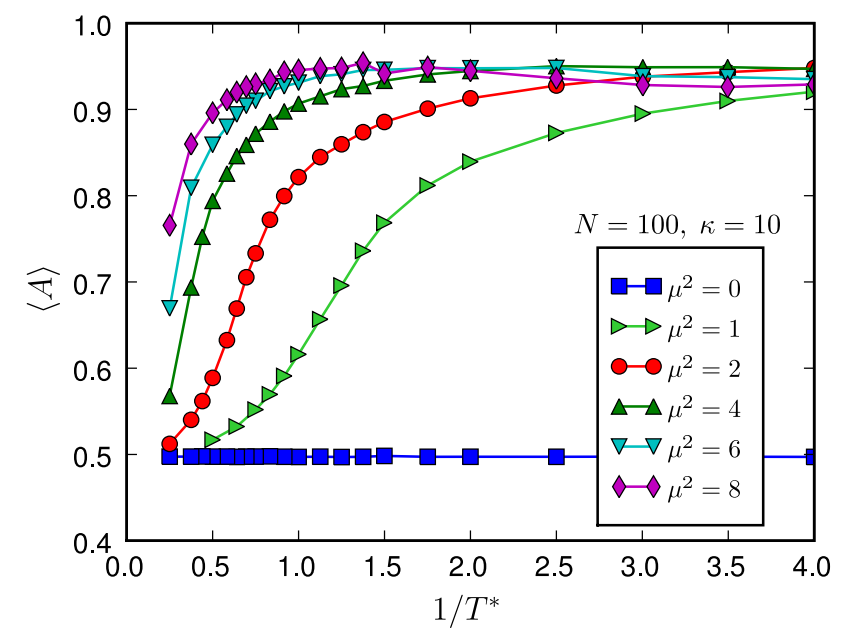

Fig. 3 Bond-dipole local orientation parameter as a function of the inverse temperature for different values of the magnetization for the case $N=100$ and $\kappa=10$.

energy $\langle\Gamma\rangle$ and the specific heat at constant volume $C_{V}$ which are defined in terms of the adsorption energy of the chain as:

$$
\begin{aligned}
\Gamma & =\left|\frac{U_{a d s}}{U_{a d s}^{(\max )}}\right|, \\
\frac{C_{V}}{N} & =\frac{\left\langle U_{a d s}^{2}\right\rangle-\left\langle U_{a d s}\right\rangle^{2}}{N T^{2}},
\end{aligned}
$$

where

$$
U_{a d s}=\sum_{i=1}^{N} U_{s}\left(z_{i}\right)
$$

and $U_{a d s}^{(\max )}$ is the largest possible adsorption energy for the chain, i.e. when all the monomers are located at the minimum of the potential $U_{a d s}^{\max }=N U_{s m}\left(z=3^{1 / 6} \sigma\right)$.

In Figure 4 we present the change of the mean normalized adsorption energy $\langle\Gamma\rangle$ as a function of the inverse temperature $1 / T$ for the case $N=100$ at different values of the dipolar strength $\mu^{2}$. Figure ?? corresponds to the stiff filament case $(\kappa=10)$. The inset plots show the behavior of the specific heat data that is derived from the energy fluctuations. The position of peak of $C_{V}$ determines the characteristic adsorption temperature at which the filament adsorbs onto the surface. Chain stiffness is observed to increase the adsorption temperature in agreement with previous works for non-magnetic chains ${ }^{37,60-62}$. We found that an increase of the dipolar interaction $\left(\mu^{2}<10\right)$ favors the adsorption at higher temperatures in a similar way that an increase of the chain stiffness does. This result can, in principle, be expected because the magnetic term reaches a local minimum when the dipoles are aligned in a parallel head to tail orientation favoring more stretched con- 


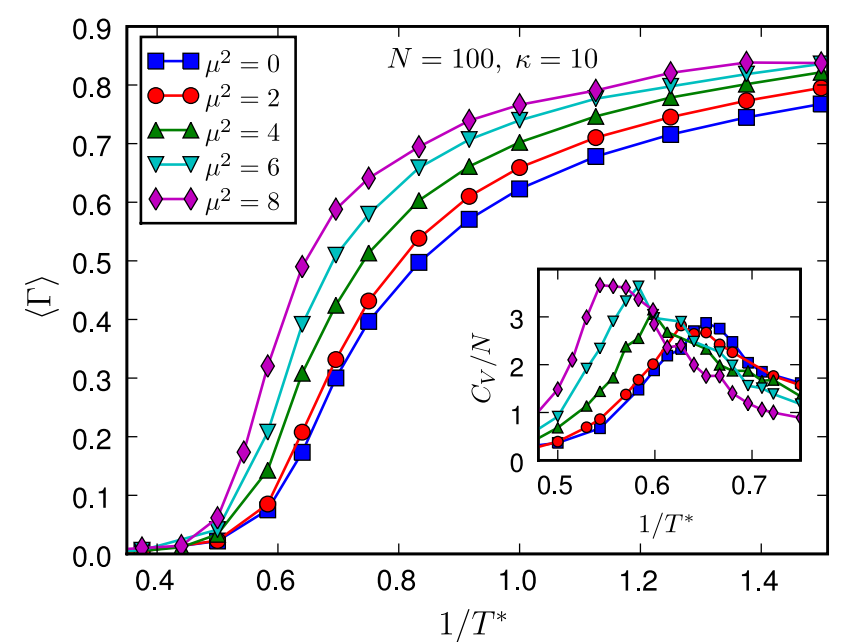

Fig. 4 Mean normalized adsorption energy, $\langle\Gamma\rangle$, and its corresponding specific heat (inset figures), as a function of the inverse temperature $1 / T$ for $N=100$ and different values of the dipolar interaction $\mu^{2}$. (a) Flexible filaments $(\kappa=0)$; (b) Stiff magnetic chains $(\kappa=10)$.

figurations. Therefore, the stiffness and the magnetic interaction promote the development of more stretched chains and, as it happens with non-magnetic polymers ${ }^{60}$, ordered chains adsorb at higher temperatures since they lose much less entropy when adsorbed onto the surface. However, at lower temperatures and moderate values of the dipolar interaction, the tendency of the chains to stretch is reversed and the equilibrium chain conformations are characterized by the formation of ordered closed loops with a circular shape. This behavior will be further analyzed in the next section.

The adsorption transition also exhibits an indication of criticality in its dependence with the system size. The adsorption takes place at higher temperatures as the chain length increases (Fig. 5(a)). For a given chain stiffness, the characteristic adsorption temperature depends on both the intensity of the magnetic interactions and the chain length $T_{c}\left(N, \mu^{2}\right)$. The critical adsorption temperature $T_{c}\left(N \rightarrow \infty, \mu^{2}\right)$ can be derived by the extrapolation of the curves by least-square fitting assuming a $T_{c}\left(N, \mu^{2}\right) \sim N^{-\phi}$ dependence, where $\phi$ is the critical crossover exponent (see Fig. 5(b)). The best fit to the data led to $\phi=0.59 \pm 0.1$ consistent with previous findings for the adsorption of homopolymers onto flat surfaces ${ }^{33,37,60}$.

In order to examine the critical adsorption properties of stiff magnetic filaments we assumed initially the scaling ansatz for non-magnetic chains ${ }^{33,37}$. Using the values of $T_{c}\left(\infty, \mu^{2}\right)$ we did a scaling analysis for the adsorption energy $U_{a d s}$ :

$$
U_{a d s} / N^{\phi}=h\left(\tau N^{\phi}\right),
$$

where $\tau$ is the relative distance to the transition point, $\tau=$

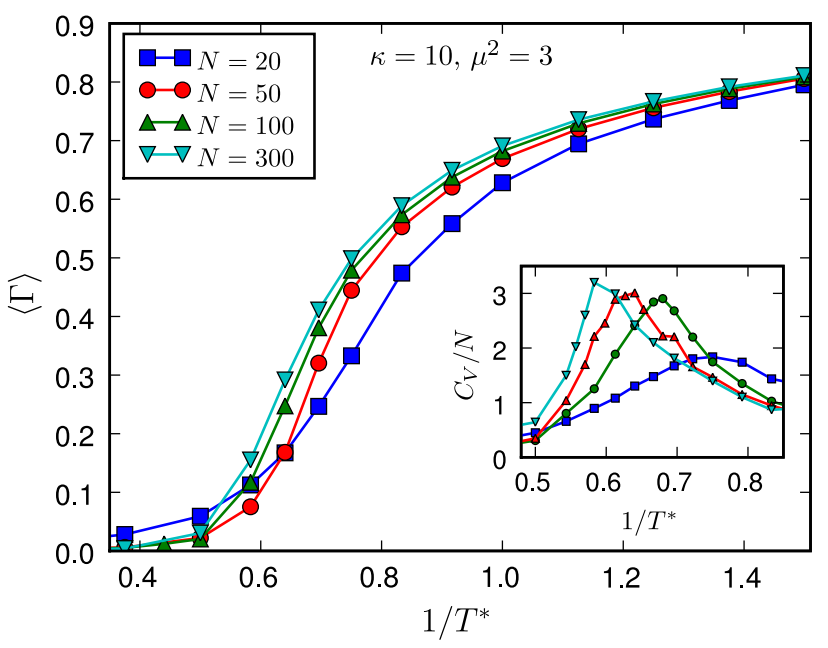

(a)

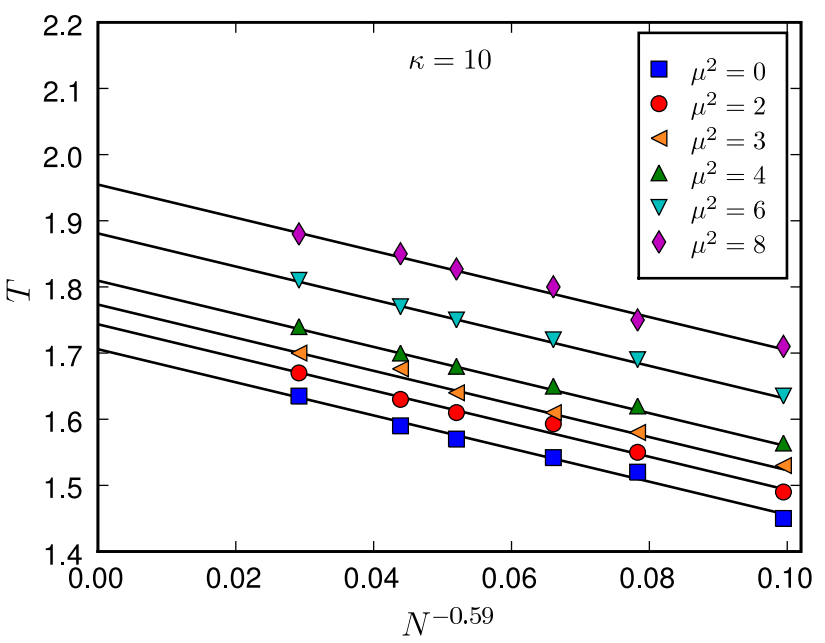

(b)

Fig. 5 (a) Same as Figure 4 for $\mu^{2}=3$ and $\kappa=10$ for different values of the chain length $N$, illustrating the finite chain size effects. (b)Determination of the critical adsorption temperatures

$T_{c}\left(N \rightarrow \infty, \mu^{2}\right)$ for different values of the dipolar interaction $\mu^{2}$. The chain stiffness is set to $\kappa=10$. The best fit to the data (solid lines) gives a crossover exponent $\phi=0.59 \pm 0.1$.

$\left(T-T_{c}\left(\infty, \mu^{2}\right)\right) / T_{c}\left(\infty, \mu^{2}\right)$, and $h(x)$ is the following scaling function $^{33}$ :

$$
h(x) \propto \begin{cases}x^{-1}, & x \rightarrow \infty \\ \text { const. }, & x \rightarrow 0 \\ |x|^{(1-\phi) / \phi}, & x \rightarrow-\infty\end{cases}
$$

Hence, the adsorption energy has the following asymptotic behavior:

$$
U_{a d s} / N \propto \begin{cases}0, & T \geq T_{c}\left(N \rightarrow \infty, \mu^{2}\right) \\ |\tau|^{(1-\phi) / \phi}, & T<T_{c}\left(N \rightarrow \infty, \mu^{2}\right)\end{cases}
$$




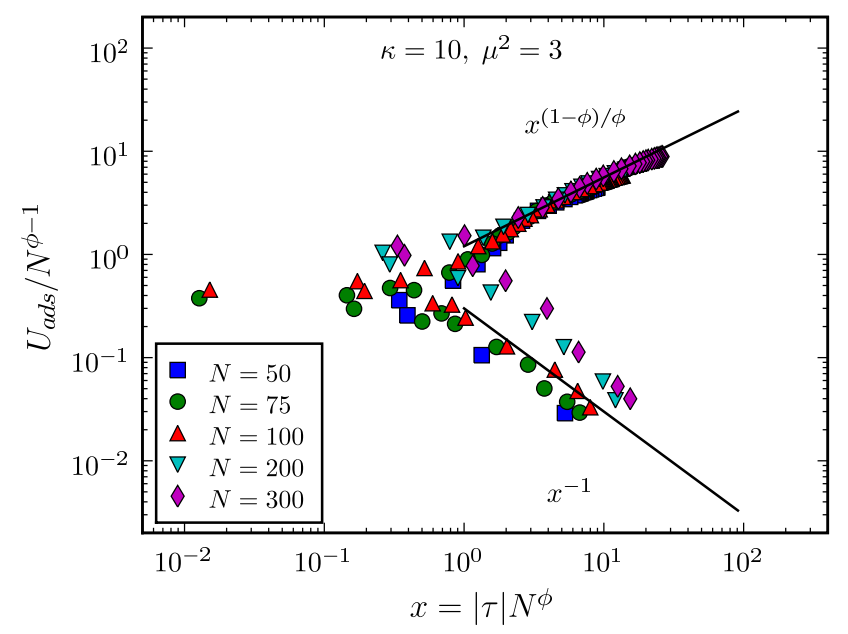

Fig. 6 The log-log plot of the scaling function $U_{a d s} / N^{\phi-1}$ vs the scaling argument $|\tau| N^{\phi}$ for the case $\kappa=10$ and $\mu^{2}=3$. The straight lines indicate the asymptotic limit. Error bars are covered by the symbols.

The results for $\mu^{2}=3$ is shown in Fig.??. Our data reproduce the conventional classical scaling relationship and confirm the value of 0.59 for the crossover exponent. Although we only report here the plot for $\mu^{2}=3$, the same agreement has been observed for all the values of the dipolar interaction used in our simulations $\left(\mu^{2}<10\right)$.

A further insight on the adsorbed chain conformations close to the adsorption transition point can be obtained from the study of the statistics of the three different types of segments that can be identified in the filament structure, namely: trains, loops and tails. A train is defined as a sequence of consecutive monomers where all them are adsorbed onto the surface, i.e. $z_{i}<z_{\text {cut }}$. A sequence of consecutive monomers where no one is adsorbed, i.e. $z_{i}>z_{\text {cut }}$ defines a loop if both edges are bounded to train-type monomers, otherwise when only one of the edges is connected it defines a tail. An schematic representation of these motifs is depicted in Fig. 7(a).

Recently, the statistics of loops, trains and tails in the detachment process of a single chain from a sticky substrate when the chain is pulled by an external force has been theoretically analyzed by Bhattacharya et al. ${ }^{83}$. The results we found in the case of a stiff magnetic filament near an attractive surface are summarized in Fig.7(b). In this figure we represent the averaged number of monomers involved in loops, trains or tail structures $\langle n\rangle$ as a function of $1 / T$. We observe a monotonous increase in the averaged number of monomers in train segments as the temperature reduces, mimicking the behavior of the adsorption energy $\langle\Gamma\rangle$ during the adsorption. This process is accompanied by a monotonous decrease of monomers in tails, as expected. The number of monomers

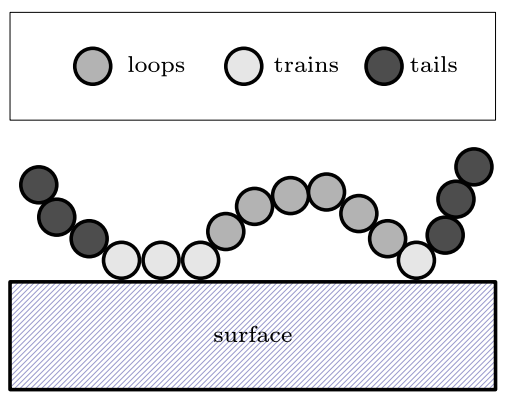

(a)

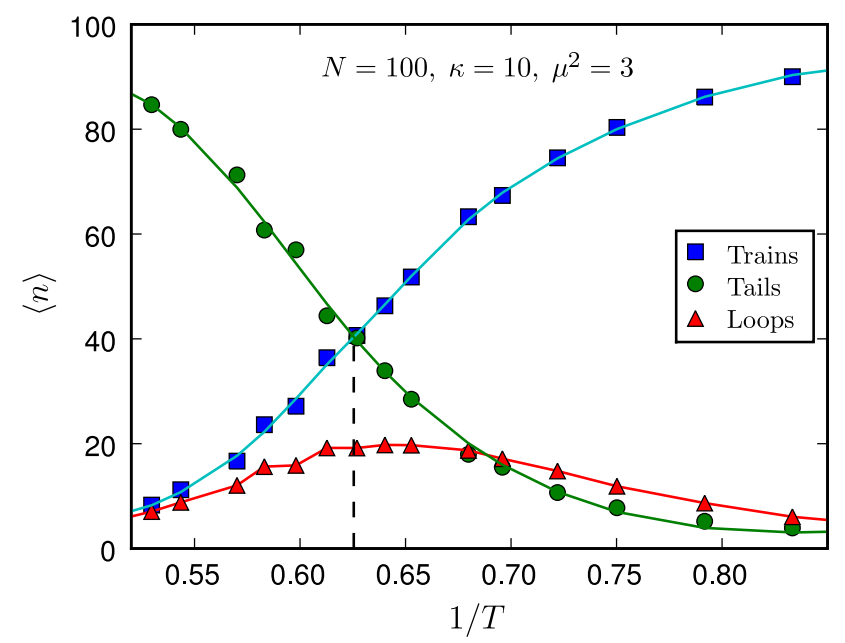

(b)

Fig. 7 (a) Schematic representation of the different types of filament structures developed close to the adsorption transition point. (b) Average number of monomers involved in train, tail and loop segments close to the adsorption transition point for $N=100$, $\kappa=10$ and $\mu^{2}=3$. The dashed line identifies the temperature at the train-tail crossing point, $T_{t t}$. The error bars are covered by the size of the symbols.

involved in loops reaches a maximum close to the characteristic adsorption transition temperature. This behavior is consistent with the idea that close to the transition point the adsorbed/unadsorbed fluctuations should be maximal. Nonetheless, the location of this maximum is slightly shifted to smaller temperatures with respect to the transition temperature derived from the position of the peak in the specific heat. Interestingly, we found that the temperature at which the curves corresponding to the number of monomers involved in trains and tails intersect, namely $T_{t t}$, is strongly correlated with the characteristic adsorption temperature $T_{c}\left(N, \mu^{2}\right)$ as shown in Figure 8. Within a precision of $\Delta T= \pm 0.04$ both temperatures coincide for all the cases studied. To our best knowledge, this is the first time in which a technique based on the analysis of the train and tail statistics is used to determine the characteristic 


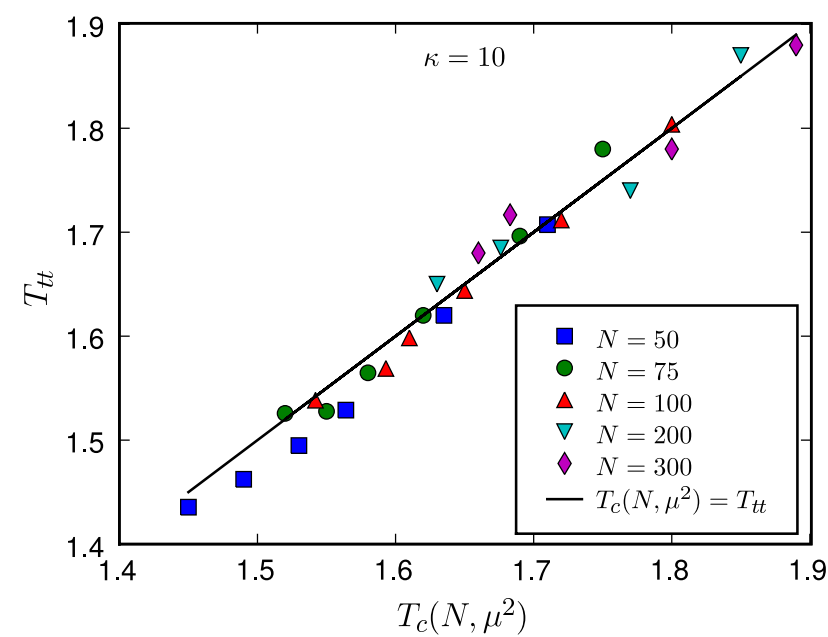

Fig. 8 Correlation between the characteristic temperatures derived from the position of the peak in the specific heat data $T_{c}\left(N, \mu^{2}\right)$ and the intersection point between the averaged number of monomers in train and tail segments $T_{t t}$ for $\kappa=10, \mu^{2}=0,2,3,4,6,8$ and different chain lengths $N$.

adsorption temperature. In addition, this technique has the advantage that good estimates can be obtained with much less numerical effort.

A usual approach to characterize the filament conformations is the study of the behavior of the parallel, $\left\langle R_{g, \|}^{2}\right\rangle^{1 / 2}$, and perpendicular, $\left\langle R_{g, \perp}^{2}\right\rangle^{1 / 2}$, components of the root mean squared radius of gyration $\left\langle R_{g}^{2}\right\rangle^{1 / 2}$ as a function of $1 / T$. The results for the case $N=100$ and $\kappa=10$ and different values of the dipolar strength $\mu^{2}$ are shown in Figure 9. The perpendicular component drops to zero as soon as the chain becomes adsorbed onto the surface as previously observed in non-magnetic polymer chains ${ }^{60}$. More interesting is the analysis of the parallel component of the radius of gyration. In non-magnetic filaments, as the thermal noise reduces with the temperature, the stiffness component dominates and more stretched configurations are developed. As a consequence, we expect an increase in the parallel component of $R_{g}$ as it is clearly illustrated in Figure 9 for $\mu^{2}=0$. However, as soon as we turn on the dipolar interaction such monotonous growth reverts at a given temperature, that depends on $\mu^{2}$, and $\left\langle R_{g, \|}^{2}\right\rangle^{1 / 2}$ starts to decrease. This new behavior, characteristic of the magnetic filaments, is in agreement with the previous observation that at low temperatures and moderate values of the dipolar interaction $\left(\mu^{2}<10\right)$, the equilibrium chain conformations are characterized by the presence of closed structures in the adsorbed state. This result suggests the existence of a second structural transition that takes place at temperatures below $T_{c}\left(\infty, \mu^{2}\right)$.

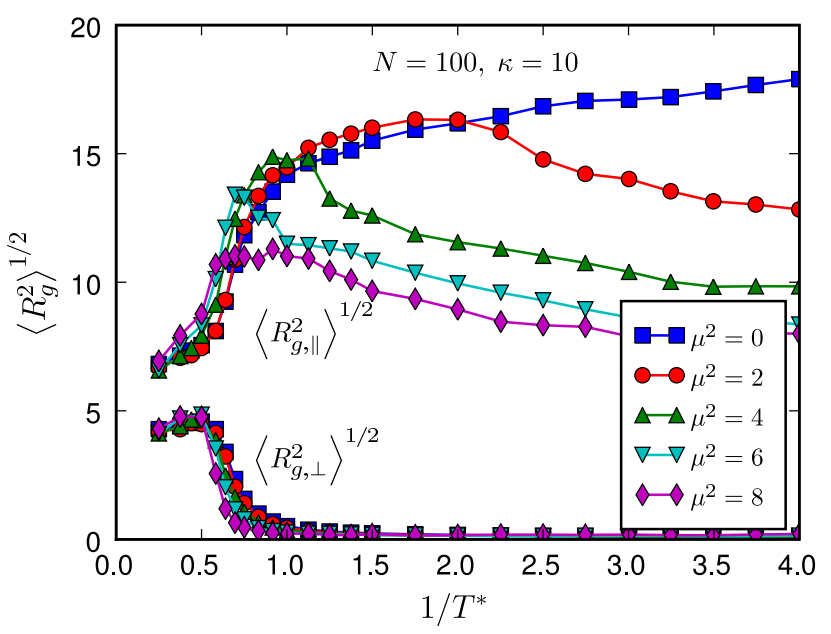

Fig. 9 Parallel, $\left\langle R_{g, \|}^{2}\right\rangle^{1 / 2}$, and perpendicular, $\left\langle R_{g, \perp}^{2}\right\rangle^{1 / 2}$, components of the root mean squared radius of gyration as a function of $1 / T$ for a magnetic filament with $N=100, \kappa=10$ and different values of the dipolar interaction $\mu^{2}$.

\subsection{The structural open-closed transition}

Recent studies in quasi-two dimensional ferrofluid systems ${ }^{73}$ showed that the ground state conformations correspond to annular structures. Such ferrofluid particles are observed to assemble in one-dimensional chains in the presence of magnetic interactions ${ }^{16}$. Thus, a major difference between a ferrofluid system and magnetic filaments is the presence, in the latter, of a bending potential responsible for the chain stretching. Therefore, depending on the system temperature, the following scenarios are expected to develop for magnetic filaments: At high temperatures, thermal fluctuations dominate and an unadsorbed random coil is expected. At smaller temperatures, the bending potential together with the dipolar interactions favor more ordered chains that adsorb onto the surface. At this stage, the interaction between neighbour dipoles dominates over the long range contribution of distant monomers. Since the magnetic term reaches a local minimum when dipoles are aligned in a parallel head to tail orientation more stretched chains are favored. However, when temperature is further reduced, the long range magnetic interaction dominates and ring-like structures prevail over stretched conformations.

In order to characterize the structural transition of the magnetic filaments in the adsorbed state, we have computed the root mean squared end-to-end distance, $\left\langle R_{e e}^{2}\right\rangle^{1 / 2}$, and the accumulated normalized spontaneous magnetization parallel and perpendicular to the attractive surface: $\langle M\rangle=$ $\left\langle\left|\sum_{i=1}^{N} \boldsymbol{\mu}_{i, \|}\right| /(N \mu)\right\rangle$ and $\langle M\rangle=\left\langle\left|\sum_{i=1}^{N} \boldsymbol{\mu}_{i, \perp}\right| /(N \mu)\right\rangle$. We expect that these functions will strongly evidence the conformal changes in the polymer chain at low temperatures. The re- 


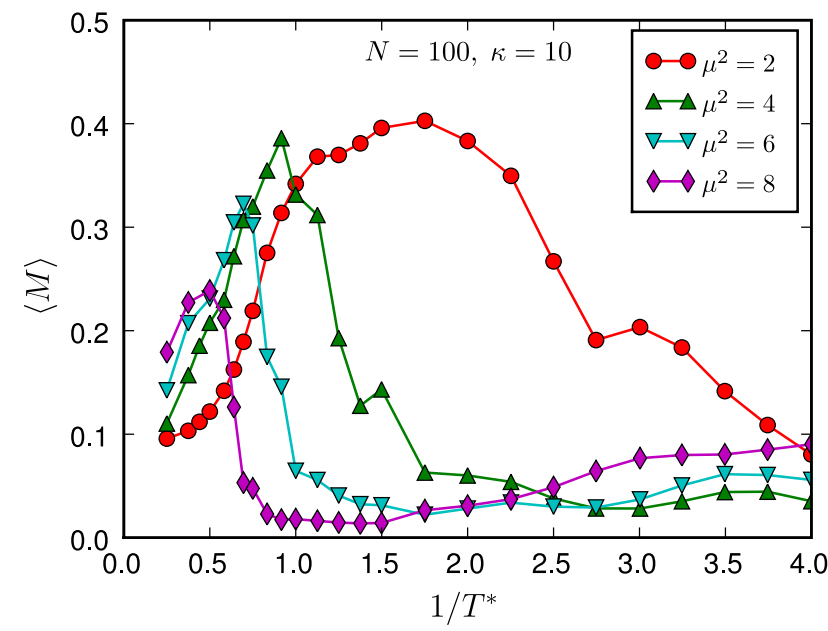

Fig. 10 Normalized parallel and perpendicular magnetization to the surface, $\left\langle M_{\|}\right\rangle$and $\left\langle M_{\perp}\right\rangle$ respectively, as a function of $1 / T$ for $N=100, \kappa=10$ for non-zero values of the dipolar interaction $\mu^{2}$.

sults of the end-to-end distance $\left\langle R_{e e}^{2}\right\rangle^{1 / 2}$ and its parallel and perpendicular components are observed to follow closely the behaviour found for the $\langle M\rangle$ and its parallel and perpendicular components. Figure 10 shows $\left\langle M_{\|}\right\rangle$and $\left\langle M_{\perp}\right\rangle$ as a function of $1 / T$ for $N=100$ and $\kappa=10$. The perpendicular component reduces to zero after the adsorption transition, while $\left\langle M_{\|}\right\rangle$displays a maximum which value and position depends on the dipole modulus of the magnetic moments. The similar evolution of the parallel components of the end-to-end distance and magnetization confirms the idea that moderated magnetic interactions initially tend to stretch the chain during the adsorption process. For non zero dipolar strengths, and after reaching a maximum value, $\left\langle R_{e e}^{2}\right\rangle^{1 / 2}$ and $\langle M\rangle$ drops almost to zero as a clear indication that a closed structure is being formed. This process operates at higher temperatures as the dipolar strength increases. The averaged accumulated parallel magnetization $\langle M\rangle$ (Fig.10) exhibits the same type of behavior observed in $\left\langle R_{e e}^{2}\right\rangle^{1 / 2}$ with an almost one to one correspondence in filaments sharing identical system parameters. This result is a direct consequence of the observed local alignment of the dipoles with the chain backbone (Fig.3). If a perfect alignment should exist, the magnetization vector $\mathbf{M}$ and the end-to-end vector $\mathbf{R}_{\mathbf{e e}}$ would only differ by a constant factor.

The dependence of the end-to-end distance with the filament length and its fluctuations, at a fixed dipolar strength, is presented in Figure 11. In analogy to the observed behavior of the fluctuations in the adsorption energy (see Fig. 5(a)), a maximum in the fluctuations can also be observed here, and the position of this maximum shifts towards higher temperatures as the chain length increases. This behavior is a clear signature of the existence of a new transition, and the position

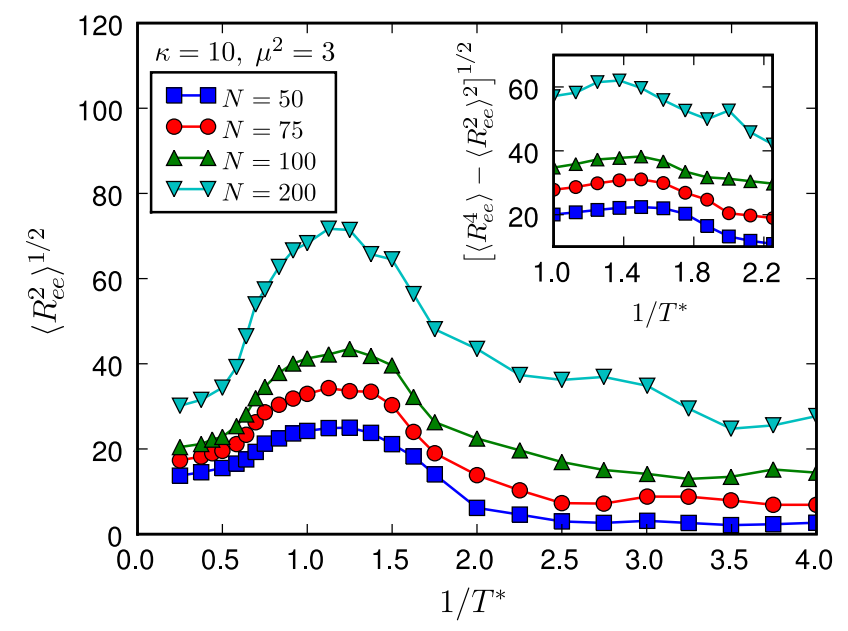

Fig. $11\left\langle R_{e e}^{2}\right\rangle^{1 / 2}$ vs. $1 / T$ for $\kappa=10, \mu^{2}=3$ and different values of the filament length $N$. Inset: fluctuation of the chain end-to-end distance.

of the maximum in the fluctuations characterizes the transition temperature from an open to closed filament structure. In order to estimate the critical temperature for this new transition we proceed in a similar way as the one we followed for the adsorption energy. For each value of the intensity of the dipolar strength $\mu^{2}$, we assume that the measured characteristic temperatures follow a power law dependence with the chain length $T_{c}\left(N, \mu^{2}\right)=a-b N^{-\psi}$. Our best fit to the data provides a crossover exponent $(\psi=0.7 \pm 0.1)$, though estimated temperatures are quite insensitive to the value of $\psi$ within the error range. The critical temperature can be obtained extrapolating the curves in the limit $N \rightarrow \infty$.

Based on the results obtained for the critical adsorption and the structural transition temperatures we plot in Figure 12 a phase diagram for semiflexible magnetic filaments near an attractive homogeneous surface for moderate values of the dipolar interaction $\left(\mu^{2}<10\right)$. We can identify three different regions. At high temperatures chains remain in the unadsorbed state for any value of $\mu^{2}$. At intermediate temperatures there exist a region where the magnetic chains are adsorbed an exhibit preferentially open structures. At smaller temperatures, beyond a minimal dipolar strength, another phase is identified where chains with closed loop structures are favored.

The phase diagram for values of $\mu^{2}>10$ and/or temperatures $1 / T>>4$ remains still to be investigated. For strong dipolar interactions $\left(\mu^{2}>10\right)$ compared to the strength of the adsorption potential $\varepsilon_{s}$, we expect the existence of partially adsorbed compact globular states. At very low temperatures adsorbed ground state structures that minimizes the dipolar and stiff contributions are expected to develop. 


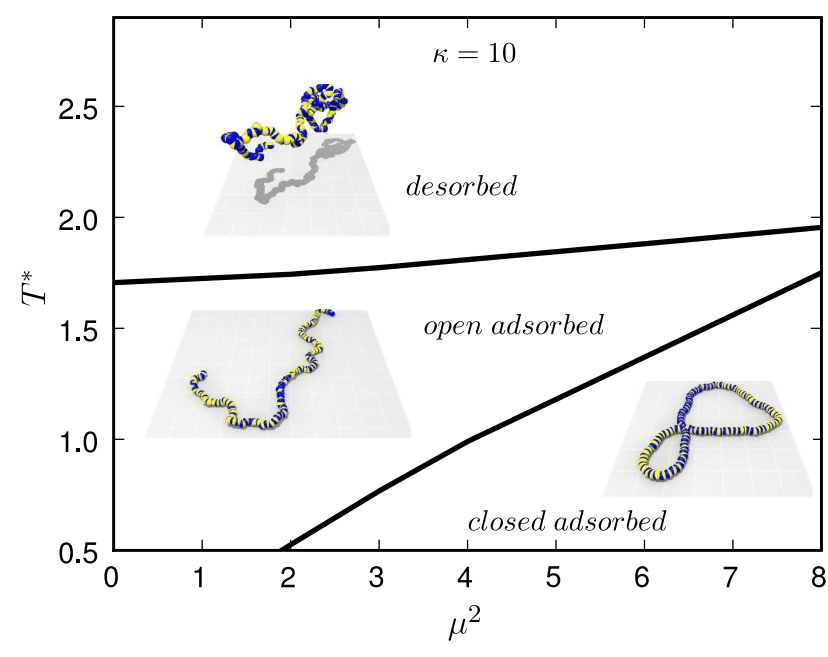

Fig. 12 Plot of the phase diagram of a single stiff magnetic filament $(\kappa=10)$ near an attractive surface for moderate values of magnetization $\left(\mu^{2}<10\right)$.

\section{Conclusions}

In the present work we have studied the behavior of stiff magnetic filaments in the neighborhood of an attractive homogeneous surface. For moderated values of the temperature $(1 / T<4)$, and dipolar magnetic interactions not large enough to induce partially desorbed states $\left(\mu^{2}<10\right)$, we found the magnetic filaments to exhibit two different transitions. The first one is the well known continuum adsorption transition. At this stage our results indicate that the dipolar interaction plays a similar role to the chain stiffness due to the tendency of the chain to stretch in order to favor the alignment head to tail of the dipoles. Thus, magnetic filaments are observed to adsorb at higher temperatures with respect to similar chains without magnetic interactions.

A novel technique to determine the characteristic adsorption temperature based on the intersection of the curves associate to the averaged number of monomers participating in trains and tails respectively has been established. The new technique offers results that are in agreement to the ones obtained with the analysis of the position of the peak in the specific heat data, but has the advantage that better estimates can be obtained with much less computational effort.

A further scaling analysis of the adsorption transition for magnetic filaments reveals that the scaling exponents are similar to those derived for non magnetic flexible and semiflexible polymer chains. Therefore, one can conclude that the adsorption transition is only affected by the magnetic interactions via a reduction of the entropy associated to the chain conformations when the dipoles force more stretched structures.

In contrast to the case of non-magnetic chains, magnetic fil- aments are observed to exhibit a second structural transition once in the adsorbed state. The transition leads to an inversion in the tendency of the magnetic chains to stretch as the temperature is reduced. Below a certain characteristic temperature the magnetic chains tend to form adsorbed closed loop structures. This change in the behavior of the magnetic chains is evidenced by a decrease in the parallel component of the radius of gyration, the mean end-to-end distance and the accumulated chain magnetization.

A closer analysis of the fluctuations of the end-to-end distance evidences the existence of a maximum value whose position identifies a characteristic transition temperature from an open to a closed filament structure. The temperature for this second structural transition is observed to increase with the chain length and the strength of the dipolar interactions.

As for the case of ferrofluid particles that are known to form annular structures in quasi-two dimensional geometries, one would expect that the ground state of a magnetic filament corresponds to structures forming almost perfect circular rings with an optimal size. This ground state is achieved at system temperatures small enough for the long range dipolar interaction to prevail over the chain stiffness.

A representation of the phase diagram for stiff magnetic filaments close to an attractive surface is also reported. The analysis of the behavior at very low temperatures and/or with strong dipolar interactions, that is left for future work, will enrich the present picture with new phases, such as, partially adsorbed globular structures.

The present work is just a first step towards the understanding of the behavior of magnetic filaments near an attractive surface but an step that we hope it will stimulate further developments on the subject of increasing scientific interest represented by this type of systems.

\section{Acknowledgements}

J.J. Cerdà and C. Holm acknowledge support by the DFG grant HO 1108/12-2 and the Volkswagen foundation. T. Sintes acknowledges Project No. FIS2007-60327 funded by the Spanish MEC. 